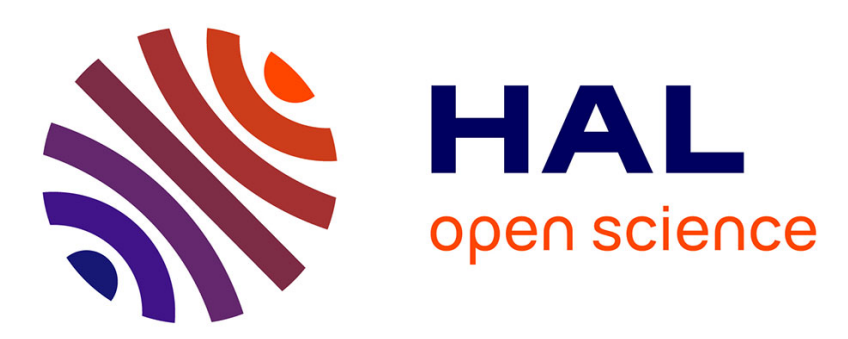

\title{
Variational segmentation of vector-valued images with gradient vector flow
}

Vincent Jaouen, Paulo Gonzalez, Simon Stute, Denis Guilloteau, Sylvie

Chalon, Irène Buvat, Clovis Tauber

\section{- To cite this version:}

Vincent Jaouen, Paulo Gonzalez, Simon Stute, Denis Guilloteau, Sylvie Chalon, et al.. Variational segmentation of vector-valued images with gradient vector flow. IEEE Transactions on Image Processing, 2014, pp.1. hal-01064937

\section{HAL Id: hal-01064937 \\ https://hal.science/hal-01064937}

Submitted on 17 Sep 2014

HAL is a multi-disciplinary open access archive for the deposit and dissemination of scientific research documents, whether they are published or not. The documents may come from teaching and research institutions in France or abroad, or from public or private research centers.
L'archive ouverte pluridisciplinaire HAL, est destinée au dépôt et à la diffusion de documents scientifiques de niveau recherche, publiés ou non, émanant des établissements d'enseignement et de recherche français ou étrangers, des laboratoires publics ou privés. 


\title{
Variational segmentation of vector-valued images with gradient vector flow
}

\author{
Vincent Jaouen, Student Member, IEEE, Paulo González, Simon Stute, Denis Guilloteau, Sylvie Chalon, \\ Irène Buvat, Senior Member, IEEE, and Clovis Tauber*, Member, IEEE
}

\begin{abstract}
In this paper, we extend the gradient vector flow field for robust variational segmentation of vector-valued images. Rather than using scalar edge information, we define a vectorial edge map derived from a weighted local structure tensor of the image that enables the diffusion of the gradient vectors in accurate directions through the 4DGVF equation. To reduce the contribution of noise in the structure tensor, image channels are weighted according to a blind estimator of contrast. The method is applied to biological volume delineation in dynamic PET imaging, and validated on realistic Monte Carlo simulations of numerical phantoms as well as on real images.
\end{abstract}

Index Terms-Deformable models, Dynamic PET, Gradient vector flow, Structure tensor

Abstract-In this paper, we extend the gradient vector flow field for robust variational segmentation of vector-valued images. Rather than using scalar edge information, we define a vectorial edge map derived from a weighted local structure tensor of the image that enables the diffusion of the gradient vectors in accurate directions through the 4DGVF equation. To reduce the contribution of noise in the structure tensor, image channels are weighted according to a blind estimator of contrast. The method is applied to biological volume delineation in dynamic PET imaging, and validated on realistic Monte Carlo simulations of numerical phantoms as well as on real images.

Index Terms-Deformable models, Dynamic PET, Gradient vector flow, Structure tensor

\section{INTRODUCTION}

\section{A. Motivation}

$\mathbf{V}$ ECTOR-valued images occur in contexts such as color images, images of the same field of view acquired at different wavelengths (i.e. multi and hyper spectral images), medical images acquired at different time intervals (e.g. dynamic PET or functional MR images), with different equipments (e.g. coregistered PET/CT images), different modes (e.g. multi-spin echo MR images) or textured images. The accurate segmentation of these images require appropriate methods for exploiting additional information provided by the extra dimension [1]-[5].

Deformable models such as snakes, as originally proposed by Kass and Witkin [6] and active surfaces in 3D have become very popular in image segmentation, including for medical

*Asterisk indicates corresponding author.

Copyright (c) 2013 IEEE. Personal use of this material is permitted. However, permission to use this material for any other purposes must be obtained from the IEEE by sending a request to pubs-permissions@ieee.org.

V. Jaouen, P. González, D. Guilloteau, S. Chalon and C. Tauber* are with Université François-Rabelais de Tours, INSERM, UMR U 930 "Imaging and Brain" unit, Hôpital Bretonneau, Tours, France

Simon Stute and Irène Buvat are with CEA, I2BM, SHFJ, Orsay, France. applications [7]. Active surface models attempt to recover the region of interest by conforming an evolving surface to the boundary of the object. The evolution of the surface is derived through a variational formulation of an energy functional which can be seen, when reaching equilibrium, as a force-balance relation between forces acting on the surface: internal forces, which control the smoothness of the model, and external forces, derived from image information. Such external forces can either use local information like edges [6], [8]-[10], global information based on region statistics [11], hybrid approaches based on both local and global information [12]-[15] or based on edge pixels interactions [16].

Implicit representations of deformable models such as the geometric active contour model are able to handle topological changes through the level-set paradigm [17], [18]. This topological flexibility can however constitute a drawback when a single object has to be segmented, as it can be the case in medical image segmentation. In such cases, additional topology-preserving procedures must be implemented [19], [20]. Another drawback of implicit representations is the increased computational load, which can be prohibitive in 3D clinical imaging. On the other hand, parametric deformable models are particularly appropriate for single object delineation because of their inherent ability to preserve the topology of the initial model. In this study, we focus on parametric edge-based deformable models.

Efforts have been made to overcome the original limitations of parametric snakes, mainly through the derivation of new expressions for the external force field that guides the model toward the boundaries of the object [8]-[10], [21]-[23]. Among external force fields, the Generalized Gradient Vector Flow (GGVF) field [8], [9], and more recently the Vector Field Convolution (VFC) field [10] aroused great interest because of their reduced sensitivity to noise and their ability to progress into highly concave regions in the image, the latter property being due to their nonconservative nature. However, the efficiency of both GGVF and VFC force fields critically relies on the choice of an accurate scalar edge map, usually based on the spatial derivatives of the input image, e.g. a Canny filter [24]. Refinements of these methods have been proposed to tackle remaining issues such as sensitivity to initialization [25], [26], capture range and ability to move into long and thin concavities [27].

Aside from these improvements, deformable models tailored for vector-valued images either using edge-based or regionbased approaches have been proposed [3], [13], [28]-[30]. In the pioneering work of Di Zenzo [31], the image is considered 
as a vector field whose dimension is the number of channels in the image. Edge detection is associated with the magnitude of the gradient of the vector-valued image, or vector gradient, derived from the norm of a local structure tensor (LST) that integrates the different gradient contributions to locate real edges, or vector edges more precisely. Structure tensors, also known as second moment matrices, can estimate magnitudes and directions of oriented structures like edges at a local scale. They have been widely studied, especially in the field of image restoration [5], [32]-[34]. In particular, Tschumperlé and Deriche have devised a generic PDE (partial differential equations)-based formulation for the regularization of vectorvalued images that exploits both amplitudes and directions of the vector gradient computed from local structure analysis. In image segmentation, LST have been studied first by Sapiro [2], [28] for geometric active contours, who set the edge-stopping term of the level set as a function of the norm of the LST. This approach has been also used by Xie and Mirmehdi [13]. Goldenberg et al. used an alternative metric tensor based on the Beltrami framework, where the color image is considered as a two dimensional surface in a five dimensional space [29]. Zhang et al. exploited the LST formalism for seeded segmentation based on anisotropic diffusion [35]. In the case of parametric active contours, the gradient magnitude in the Luv color space was incorporated in the GVF framework by Yang et al. [30] under the name Color GVF.

While the use of directions and magnitude derived from local geometry analysis is well established for image regularization using anisotropic diffusion, to our knowledge, there is no deformable model approach that also takes profit of the directional information carried by the LST for image segmentation. Current deformable models generally identify vector edges by a simple scalar value, i.e. the norm of the LST. Another drawback of existing methods is that the different channels of the image participate equally in the calculation of the gradient, regardless of their relevance.

\section{B. Outline of present work}

The aim of this work is to propose a new external force field for parametric deformable surfaces evolving in vectorvalued images. We focus our study on imaging modalities where detection is hampered by varying contrast and noise over the channels, but where physical edge location do not vary, i.e. where regions of interest are characterized by the homogeneity of their representation over the channels. In such situations, while image edges may be ill-defined on individual channels, the combination of information along the different channels may improve their localization. We introduce a new gradient vector flow field, the 4DGVF field (Four Dimensional Gradient Vector Flow) that takes advantage of the information available in the different channels. We define a vectorial edge map which points toward the inflection points of the vector-valued profiles and which is based on a weighted local structure tensor of the image. This vectorial edge map is propagated throughout the image by the means of a nonlinear diffusion equation. By exploiting orientations and magnitudes of the vector gradient in the GVF framework, the 4DGVF model exploits more information than models in which gradient is computed according to a scalar edge map. By weighting the LST dynamically, we control the influence of the different channels to favour the ones where the features can be better detected, hence reducing sensitivity to noise.

Applied to dynamic PET imaging of the brain, our model segments the brain structures as a function of the kinetic profiles of voxels. We assess the quality of our model using realistic dynamic Monte Carlo simulations of numerical head phantoms and apply it to real PET images. We compare our results to single-channel approaches based on VFC and GGVF as well as to vector-valued approaches.

This paper is organized as follows. Section II briefly recalls the formalism of parametric active surfaces and of GGVF fields. Section III presents the proposed 4DGVF external force field. The validation setup used for the experiments is described in section IV. Results on synthetic images are presented and discussed in section $\mathrm{V}$, followed by results on PET images in section VI. Finally, a conclusion is drawn in Section VII.

\section{BACKGROUND}

In this section, we briefly describe the mathematical background of parametric active surfaces and of the GGVF of the literature.

\section{A. Active surface model}

In the continous domain, a parametric active surface $\mathbf{S}^{\tau}$ at time $\tau$ of its deformation is represented as a mapping of a bivariate parameter $(m, n)$ on a regular grid $\Omega$, superimposed on the spatial image domain:

$$
\mathbf{S}^{\tau}: \Omega=[0,1] \times[0,1] \rightarrow \mathbb{R}^{3} .
$$

More explicitely,

$$
(m, n) \rightarrow \mathbf{S}^{\tau}(m, n)=\left[x_{1}(m, n), x_{2}(m, n), x_{3}(m, n)\right]^{T} .
$$

$\mathbf{S}^{\tau}$ deforms under the influence of forces, iteratively minimizing the following energy functional:

$$
E\left(\mathbf{S}^{\tau}\right)=\iint_{m, n}\left[\mathrm{E}_{i n t}\left(\mathbf{S}^{\tau}\right)+\mathrm{E}_{e x t}\left(\mathbf{S}^{\tau}\right)\right] d m d n,
$$

where $E_{\text {int }}$ is the internal energy and $E_{\text {ext }}$ the external energy of the surface. The internal energy term imposes smoothness constraints on the surface, while the external energy term drives it toward the object of interest. Expanding the internal energy term, where the functionals of first and second order define smoothness measures, the energy can be expressed as:

$$
\begin{aligned}
E\left(\mathbf{S}^{\tau}\right)= & \iint_{m, n}\left[\alpha\left(\left\|\mathbf{S}^{\tau}{ }_{m}\right\|^{2}+\left\|\mathbf{S}_{n}^{\tau}\right\|^{2}\right)+\beta\left(\left\|\mathbf{S}^{\tau}{ }_{m m}\right\|^{2}+\right.\right. \\
& \left.\left.\left\|\mathbf{S}^{\tau}{ }_{n n}\right\|^{2}+2\left\|\mathbf{S}^{\tau}{ }_{m n}\right\|^{2}\right)+E_{\text {ext }}\left(\mathbf{S}^{\tau}\right)\right] d m d n,
\end{aligned}
$$

where coefficients $\alpha$ and $\beta$ define the weighting of internal energies acting on the surface [36]. Elasticity terms are weighted by $\alpha$, rigidity terms and resistance to twist by $\beta$. Equation (3) can be seen as a force balance equation. At equilibrium, i.e. at minimum energy, one obtain the Euler-Lagrange equation:

$$
\alpha \Delta \mathbf{S}^{\tau}-\beta \Delta^{2} \mathbf{S}^{\tau}-\nabla E_{e x t}=0,
$$


where $\nabla$ is the gradient operator and $\Delta$ is the Laplace operator. The first two terms define internal forces acting on the surface:

$$
\overrightarrow{\mathbf{F}}_{i n t}\left(\mathbf{S}^{\tau}\right)=\alpha \Delta \mathbf{S}^{\tau}-\beta \Delta^{2} \mathbf{S}^{\tau} .
$$

The third term defines external forces derived from edge information:

$$
\overrightarrow{\mathbf{F}}_{e x t}\left(\mathbf{S}^{\tau}\right)=-\nabla E_{\text {ext }},
$$

so that an equilibrium is reached at the boundaries of the object.

\section{B. Generalized Gradient Vector Flow}

The generalized gradient vector flow (GGVF) field [9] is the vector field $\overrightarrow{\mathbf{F}}_{\text {ext }}$ defined as the steady-state solution of the following vector partial differential equations:

$$
\frac{\partial \overrightarrow{\mathbf{F}}_{e x t}}{\partial t}=g(|\nabla f|) \nabla^{2} \overrightarrow{\mathbf{F}}_{e x t}-h(|\nabla f|)\left(\overrightarrow{\mathbf{F}}_{e x t}-\nabla f\right),
$$

where each spatial component of $\overrightarrow{\mathbf{F}}_{\text {ext }}$ is solved independently. $\nabla^{2}$ is the vector Laplace operator, $f$ is an edge map derived from the image typically ranging from 0 to 1 and having strong values at the edges. $g=\mathrm{e}^{-\frac{|\nabla f|}{\kappa}}$ and $h=1-g$ are two functions that control the trade-off between the first and second terms through parameter $\kappa$. The first term favors the isotropic diffusion of $\overrightarrow{\mathbf{F}}_{\text {ext }}$ where $|\nabla f|$ has low values, e.g. in homogeneous regions. The second term tends to conform the field to $-\nabla f$ in regions of strong gradients. The resulting vector field shares the desirable properties of providing a large capture range and of allowing the models to progress into narrow concavities.

\section{METHOD}

In this section, we present the 4DGVF external force field, a generalization of GGVF fields to vector-valued images.

\section{A. Weighted structure tensor of a $3 D$ vector-valued image}

In the continuous domain, we denote by $\mathbf{I}$ a $3 \mathrm{D}$ vectorvalued image consisting of $M$ channels:

$$
\mathbf{I}(\mathbf{x}, c):\left(\Omega_{s} \otimes \Omega_{c}\right) \subset \mathbb{R}^{3} \otimes \mathbb{N} \rightarrow \mathbb{R},
$$

where $\Omega_{s}$ is the $3 \mathrm{D}$ spatial domain of the image and $\Omega_{c}$ the channel dimension. $\mathbf{x}=\left(x_{1}, x_{2}, x_{3}\right) \in \Omega_{s}$ is the spatial position of the voxel. We denote by $\mathbf{I}_{k}=I(\mathbf{x}, k)$ the $k^{t h}$ channel of the image.

A natural generalization of the notion of gradient to vectorvalued images consists in averaging the different gradient contributions in individual channels. However, this approach is generally not satisfying, for example when opposite contributions cancel out. To avoid this drawback, Di Zenzo proposed a geometrical approach in which a 2D color image is considered as a $2 \mathrm{D} \rightarrow 3 \mathrm{D}$ vector field [31]. The gradient is then identified as the direction that maximizes the quadratic form of the total differential $d \mathbf{I}$ of I. Further developments of Di Zenzo's approach have expressed this maximization problem in tensor notation [37], by introducing the structure tensor of a vectorvalued image. In the $3 \mathrm{D}$ case, $\mathbf{I}$ is a $3 \mathrm{D} \rightarrow \mathrm{MD}$ vector field and its total differential is expressed as:

$$
d \mathbf{I}=\frac{\partial \mathbf{I}}{\partial x_{1}} d x_{1}+\frac{\partial \mathbf{I}}{\partial x_{2}} d x_{2}+\frac{\partial \mathbf{I}}{\partial x_{3}} d x_{3} .
$$

A weighted quadratic form for $d \mathbf{I}$, or first fundamental form, is expressed as:

$$
\|d \mathbf{I}\|_{\omega}^{2}=d \mathbf{x}^{T} \mathbf{G}_{\omega} d \mathbf{x}
$$

with $G_{\omega}$ a regularized, weighted structure tensor of the image:

$$
G_{\omega}=K_{\sigma} * \sum_{k=1}^{M} \omega_{k}\left(\nabla \mathbf{I}_{k} \otimes \nabla \mathbf{I}_{k}^{T}\right),
$$

where $K_{\sigma}$ is a Gaussian kernel of scale $\sigma, *$ is the convolution operation, $\otimes$ is the tensor product, and $\omega_{k}$ is a weighting factor for channel $\mathbf{I}_{k}$. The weighting factors $\omega_{k}, k \in\{1 . . M\}$ characterize the reliability of the different channels.

The contribution of this article is twofold. First, we establish a new weighting strategy tailored for deformable models and that is well adapted to vector-valued images in which the representativeness of the studied object is varying along the channels. Second, we exploit the above-mentioned geometrical framework to define a new gradient vector flow field through nonlinear diffusion of both directional and scalar information carried by the LST.

\section{B. Weights calculation}

Without a priori knowledge, the extension of Di Zenzo's approach to $3 \mathrm{D}$ consists in weighting all channels equally:

$$
\omega_{\mathbb{1}}=1 / M .
$$

With such weights, contributions from noise in channels where the object is poorly represented might hamper edge detection. Different application-specific solutions have been proposed to weight the contribution of the LST, based for example on noise estimations in the diffusion framework [38], or local saliency for image fusion purposes [39].

In GVF-based approaches, it is desirable to maximize the contrast-to-noise ratio (CNR) of the gradient signal in order to perform accurate diffusion of the edge map gradient vectors throughout homogeneous regions of the image. In low contrast images, the gradient signal due to noise can be superior to the gradient signal due to true edges. This can bias the estimation of directions and magnitudes of vector edges and, consequently, the directions of the GVF force field. Here, we propose to exploit the active contours framework to define a new weighting strategy tailored for image segmentation, based on a blind estimation of the contrast of the object in each channel. Such a global weighted averaging scheme can maintain high contrast of the vector gradient signal while reducing its variance [40].

For each channel $\mathbf{I}_{k}$, let $R_{k}^{i n}$ be the set of voxels located inside $\mathbf{S}^{t}$, and let $R_{k}^{\text {out }}$ be the set of voxels located outside $\mathbf{S}^{t}$ and inside $\lambda \mathbf{S}^{t}$, a morphological dilation of $\mathbf{S}^{t}$ of $\lambda$ units of length. The limitation of $R_{k}^{\text {out }}$ to $\lambda \mathbf{S}^{t}$ prevents possible 


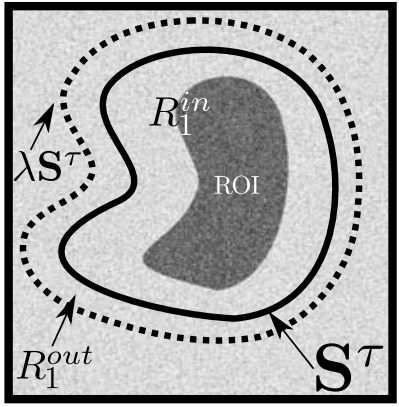

(a)

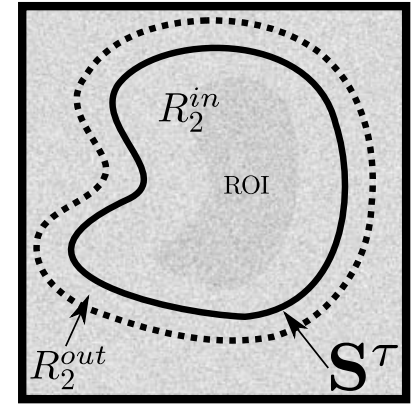

(b)
Fig. 1: Illustration of the weighting method on a $2 \mathrm{D}$ representation of the active surface $\mathbf{S}^{t}$ around a region of interest (ROI). (a) High contrast channel. (b) Low contrast channel. A measure of contrast is established by comparing average intensities in $R_{k}^{\text {in }}$ and $R_{k}^{\text {out }}$ in each channel

influence from further regions. We define the weighting factor for channel $\mathbf{I}_{k}$ as follows:

$$
\omega_{k}:=\left(\frac{\left|\overline{\mathbf{I}}_{k}^{\text {in }}-\overline{\mathbf{I}}_{k}^{\text {out }}\right|^{\gamma}}{\sum_{j}\left|\overline{\mathbf{I}}_{j}^{\text {in }}-\overline{\mathbf{I}}_{j}^{\text {out }}\right|^{\gamma}}\right),
$$

where summation is over the $M$ channels. $\overline{\mathbf{I}}_{k}^{\text {in }}$ and $\overline{\mathbf{I}}_{k}^{\text {out }}$ are the average intensities in $R_{k}^{i n}$ and $R_{k}^{\text {out }}$ respectively. $\gamma$ is a parameter that controls the linearity of the influence of the channels.

Figure 1 illustrates the weighting scheme on a conceptual 2D example. Figure 1a displays a high contrast channel where average intensities $R_{1}^{\text {in }}$ and $R_{1}^{\text {out }}$ are significantly different and for which the corresponding weight would be large. On the contrary, Figure 1b shows a low contrast channel where the corresponding weight would be low.

With such weighting factors, gradients from low contrast channels have negligible influence on the calculation of the LST, while avoiding the need for prior knowledge of the number of relevant channels. Such blind weighting scheme provides a compromise between high contrast (through weighting) and low noise (through averaging) of the vector gradient signal.

\section{Vectorial edge map}

The eigenvalues $\lambda^{+}>\lambda_{1}^{-}>\lambda_{2}^{-}$of the LST $G_{\omega}$ give the scalar rates of change of the first fundamental form in a local basis of extremal variations. Depending on the applications, these eigenvalues can be combined to define different LST norms [33], [37], [38], [41]. In our study, we choose a coherence norm proposed by Weickert in [38] that measures the amount of local anisotropy, a generalization to the 3D case of the norm presented by Sapiro in [33].

$$
\mathbf{N}_{\omega}{ }^{2}=\sqrt{\left(\lambda^{+}-\lambda_{1}^{-}\right)^{2}+\left(\lambda^{+}-\lambda_{2}^{-}\right)^{2}+\left(\lambda_{1}^{-}-\lambda_{2}^{-}\right)^{2}} .
$$

Its square root $\mathbf{N}_{\omega}$ is homogeneous to the amplitude of vector edges.

Sapiro exploited the LST formalism in an extension of the geometric active contours to 2D color images [2]. He proposed

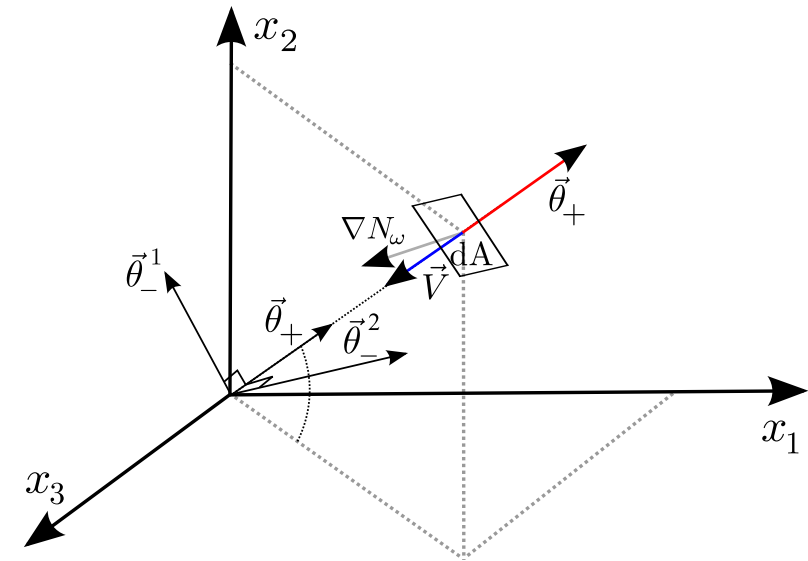

Fig. 2: Representation of a local isosurface element $d A$ of a vector-valued image. Eigenvectors of the LST $G_{\omega}$ form a local orthogonal basis of extremal variations. $\theta_{+}$is in the direction of maximum rate of change of the first fundamental form. The proposed vectorial edge map $\overrightarrow{\mathbf{V}}$ points toward vector edges and is more accurate compared to $\nabla \mathbf{N}_{\omega}$

that the edge-stopping term of the level set function be a function of the norm of a non-weighted LST, denoted here by $\mathbf{N}_{\mathbb{1}}$ (as opposed to $\mathbf{N}_{\omega}$ ). In an extension of the GVF to 2D color images, Yang et al. proposed to use the $\mathbf{N}_{\mathbb{1}}$ norm as an edge map [30]. However, restricting vector edges to a scalar measure does not take profit of all information contained in the LST, whose diagonalization gives the orientations of the gradient vectors in the image.

The directions of the eigenvectors of $G_{\omega}$ give the directions of local extrema of the quadratic form (9). The eigenvector $\vec{\theta}_{+}$ associated with the maximum eigenvalue $\lambda_{+}$gives the gradient direction, and the other two define orthogonal "isophote" directions. We thus propose here, rather than only exploiting the eigenvalues of $G_{\omega}$, to take also advantage of the directional information carried by $\vec{\theta}_{+}$in order to produce the external force field. We define a vectorial edge map $\overrightarrow{\mathbf{V}}$, a vector field collinear to the local dominant eigenvectors of $G_{\omega}$, but oriented toward the nearest vector edge:

$$
\overrightarrow{\mathbf{V}}=\vec{\theta}_{+} \operatorname{sign}\left\langle\vec{\theta}_{+}, \nabla \mathbf{N}_{\omega}\right\rangle,
$$

where $\langle$,$\rangle denotes the dot product. Figure 2$ shows a local isosurface element $d A$ in a vector-valued image. The eigenvectors of $G_{\omega}$ form an orthogonal set in the directions of maximal (gradient) and minimal (isophote) change. This illustrates the fact that the vectorial edge map $\overrightarrow{\mathbf{V}}$ is orthogonal to vector edges, which is not necessarily the case for $\nabla \mathbf{N}_{\omega}$ (and a fortiori for $\nabla \mathbf{N}_{\mathbb{1}}$ ).

\section{The $4 D G V F$ equation}

The 4DGVF external force field is the result of nonlinear diffusion of the vectorial edge map $\overrightarrow{\mathbf{V}}$ throughout the image. In the vicinity of vector edges, as detected by $\mathbf{N}_{\omega}$, the directions of the vectors are constrained by $\overrightarrow{\mathbf{V}}$, while isotropic diffusion of $\overrightarrow{\mathbf{V}}$ occurs in homogeneous regions. The 4DGVF field is 
defined as the steady-state solution of the following vector partial differential equation:

$$
\frac{\partial \overrightarrow{\mathbf{F}}_{e x t}}{\partial t}=g\left(\mathbf{N}_{\omega}\right) \nabla^{2} \overrightarrow{\mathbf{F}}_{e x t}-h\left(\mathbf{N}_{\omega}\right)\left(\overrightarrow{\mathbf{F}}_{e x t}-\overrightarrow{\mathbf{V}}\right),
$$

where $g$ and $h$ are the functions used in eq. (7), replacing the gradient magnitude $|\nabla f|$ with $\mathbf{N}_{\omega}$. At each iteration $\tau$ of the deformation, the surface $\mathbf{S}^{\tau}$ undergoes locally the external force field $\overrightarrow{\mathbf{F}}_{\text {ext }}$. To avoid convergence issues and ensure a smooth deformation, the deformation force field is projected on the normal direction to $\mathbf{S}^{\tau}$. The surface is iteratively moved according to the following gradient descent flow :

$$
\frac{\partial \mathbf{S}^{\tau}}{\partial \tau}=\alpha \Delta \mathbf{S}^{\tau}-\beta \Delta^{2} \mathbf{S}^{\tau}+\left\langle\overrightarrow{\mathbf{F}}_{e x t}, \vec{n}\right\rangle
$$

where elasticity terms are weighted by $\alpha$ and rigidity terms by $\beta . \vec{n}$ denotes the normal direction to the local surface element $d \mathbf{S}^{\tau}$. The LST is computed according to the proposed weighting scheme, and at each timestep $\tau$, weights are recomputed to construct a more accurate external force field for the next iteration.

Figure 3 shows a comparison between the eigenvector field $\vec{\theta}_{+}$(Fig. 3b) and the vectorial edge map $\overrightarrow{\mathbf{V}}$ (Fig. 3c), superimposed on one frame of a dynamic PET image (Fig. 3a). The resulting 4DGVF field is shown in figure 3d. Fields are projected on a $2 \mathrm{D}$ slice. While the diagonalization of the LST does not uniquely specify the sign of the gradient vectors, equation (14) lifts the indeterminacy and orients the field toward vector edges, a desirable property for external force fields. The resulting 4DGVF field is consistent with the studied object.

\section{E. Initialization}

Minimizing equation (3) is equivalent to finding the ideal isosurface of minimal total energy $E$. In general, the energy landscape associated with the segmentation problem is not convex, requiring the initial model to be close to the desired optimum. To this end, we propose to initialize the 4DGVF model with an extension of the Poisson Inverse Gradient (PIG) approach, proposed by $\mathrm{Li}$ and Acton [26], to vector-valued images. The PIG approach approximates the potential energy $E_{e x t}$ from which the external force field would derive. As fields such as GVF fields and VFC fields are nonconservative, this scalar potential does not exist and is estimated through a least-squares minimization problem. The initial model is identified as the isosurface of the reconstructed external energy $E_{\text {ext }}$ with lowest energy.

We adapt the PIG approach to vector-valued images and build an initialization field $\overrightarrow{\mathbf{F}}_{e x t}^{0}$ based upon the $4 \mathrm{DGVF}$ framework. We use equal weighting of all channels, following eq. (11), as finer weights such as proposed in section III-B can only be derived after an initial surface is defined. Once $\overrightarrow{\mathbf{F}}_{\text {ext }}^{0}$ is computed, we estimate the scalar potential $E_{\text {ext }}$ by solving the Poisson equation:

$$
\Delta E_{e x t}=-\nabla \cdot \overrightarrow{\mathbf{F}}_{e x t}^{0},
$$

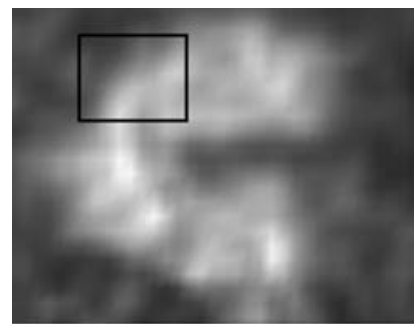

(a)

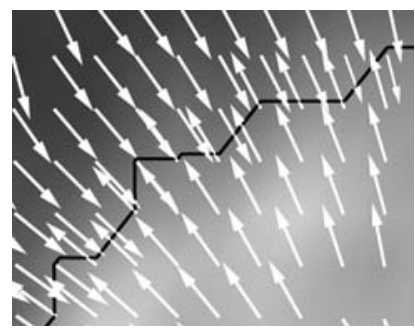

(c)

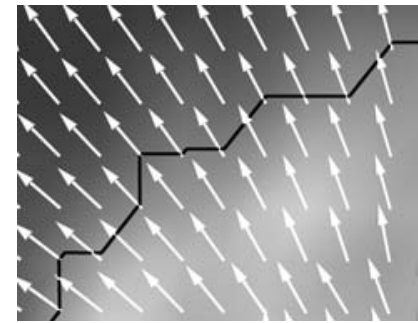

(b)

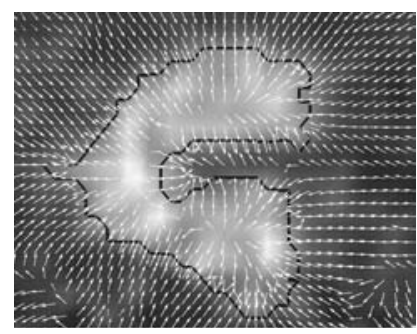

(d)
Fig. 3: (a) 2D slice of a channel of a dynamic PET image and enlarged area in (b)-(c). (b) Eigenvector field $\vec{\theta}_{+}$of the local structure tensor $G_{\omega}$. The ground truth surface is represented as a black line. (c) Vectorial edge map field $\vec{V}$ oriented toward vector edges. (d) Proposed 4DGVF field obtained by nonlinear diffusion of $\overrightarrow{\mathbf{V}}$ in the image. Vectors are mostly oriented toward closest vector edges

This equation is solved numerically by matrix inversion for which Dirichlet boundary conditions are applied on the boundary $\partial \Omega$ of the image domain $\Omega$ :

$$
E_{\text {ext }}(\partial \Omega)=-\mathbf{N}_{\omega}(\partial \Omega) .
$$

We scale $E_{e x t}$ in the range $[0,-1]$, and perform $P$ triangulated isosurface reconstructions for different values $E_{p}=$ $\left(E_{1}, E_{2} \ldots E_{P}\right), \quad E_{p} \in[0,-1]$, using a marching cubes algorithm [42]. In our experiments, we retain only closed surfaces as candidates models. We then select the surface model with minimal total external energy. This shape is then used for the computation of the initial weights prior to the deformation. To emphasize that vector-valued information is used in the initialization, we refer to this initialization in the following as Vector Poisson Inverse gradient (VPIG).

\section{F. Numerical implementation}

We implemented our method using MATLAB ${ }^{\circledR}$. The active surface $\mathbf{S}^{\tau}$ was represented as a triangulated mesh and oriented such that the normals to the faces point inwards. Normals to each vertex were computed as the weighted average of the face normals incident to the vertex [43]. We solved eq. (16) with a standard finite difference approach expressed in a matrix form [6]. The Laplacian was linearly estimated at each vertex $v_{i}$ by the umbrella operator [44]:

$$
\Delta\left(v_{i}\right)=\frac{1}{\left|i^{*}\right|} \sum_{j \in\left(i^{*}\right)} v_{j}-v_{i},
$$

where $i^{*}$ corresponds to the neighborhood of $v_{i}$ (vertices connected directly to $v_{i}$ ). As this number remained constant 
throughout the deformation and the internal forces only depended on the Laplacian, the neighborhood matrix corresponding to internal forces needed to be inverted only once. The deformable surface was considered to have converged when the maximum displacement of vertices between two iterations was less that 0.1 voxel side.

In our experiments, the amplitude maps $\mathbf{N}_{\omega}$ were scaled in the range $[0,1]$. In each image, the parameter $\kappa$ that controls the trade-off between field smoothness and gradient conformity was set so as to maximize the Jaccard similarity score between segmentation result and ground truth. The parameter $\gamma$ that controls the linearity of the weights in equation (12) was empirically set to 2 , emphasizing the relevance of high contrast channels.

\section{VALIDATION SETUP}

\section{A. Comparison with other approaches}

We compared the proposed 4DGVF approach with two single-channel and two vector-valued approaches of the literature.

- The Generalized Gradient Vector Flow (GGVF). For this single-channel approach, the diffusion of the gradient vectors is performed in each channel by finding the equilibrium solution of equation (7) [9], where the edge map of channel $\mathbf{I}_{k}$ is defined as follows:

$$
f_{k}=K_{\sigma} *\left|\nabla \mathbf{I}_{k}\right|
$$

In our experiments, we scaled every $f_{k}$ in the range $[0,1]$. The parameter $\kappa$ that controls the trade-off between field smoothness and gradient conformity was manually set so as to maximize the Jaccard similarity score between segmentation result and ground truth.

- The Vector Field Convolution (VFC) is also a single channel approach in which a convolution is performed between $f_{k}$ and a vector field kernel $\vec{C}$ whose vectors point toward the kernel's center [10]:

$$
\overrightarrow{\mathbf{F}}_{e x t}=\vec{C} * f_{k}
$$

In each image, the size of $\vec{C}$ and the power parameter of the magnitude function were set so as to maximize the Jaccard similarity score.

In our experiments, GGVF and VFC approaches were performed in each channel of the tested images. For comparison with vector-valued approaches, we retained in each $4 \mathrm{D}$ image the channel that obtained the best Jaccard score.

- The Vector Geometric Snake (VGS) is an extension of the implicit geometric snake to $3 \mathrm{D}$ vector-valued images [2], where the edge-stopping term of the level-set function is based on the gradient magnitude of the vector-valued image from a local structure tensor that weighs all channels equally:

$$
G_{\mathbb{1}}=K_{\sigma} * \sum_{k=1}^{N} \omega_{\mathbb{1}}\left(\nabla \mathbf{I}_{k} \otimes \nabla \mathbf{I}_{k}^{T}\right)
$$

The corresponding gradient magnitude $\mathbf{N}_{\mathbb{1}}$ is then derived from the eigenvalues of $G_{\mathbb{1}}$. In our experiments, we scaled $\mathbf{N}_{\mathbb{1}}$ in the range $[0,1]$.

- The Color Gradient Vector Flow (CGVF) [30] uses the gradient magnitude $\mathbf{N}_{\mathbb{1}}$ of the vector-valued image as the edge detector from which to perfom gradient diffusion in the GVF equation (7).

In our experiments, the parameter $\kappa$ was set so as to maximize the Jaccard similarity score.

Table I summarizes the edge detection terms used in the above-mentioned models.

TABLE I: Tested models and associated edge information used

\begin{tabular}{cc|cc}
\hline \hline Type & Single-channel & \multicolumn{2}{|c}{ Vector-valued } \\
Methods & GGVF,VFC & VGS,CGVF & 4DGVF \\
\hline $\begin{array}{c}\text { Edge amplitude } \\
\text { and direction }\end{array}$ & $f_{k}, \nabla f_{k}$ & $\mathbf{N}_{\mathbb{1}}, \nabla \mathbf{N}_{\mathbb{1}}$ & $\mathbf{N}_{\omega}, \overrightarrow{\mathbf{V}}$ \\
\hline \hline
\end{tabular}

\section{B. Initialization}

As results depend on the quality of the initialization, all comparative tests between models were performed using identical initialization. We generated results with the following initialization models:

- Ellipsoid initialization: an ellipsoidal shape centered around the object and fitting its shape.

- VPIG initialization: the approach described in section III-E, built upon the PIG approach and the initial 4DGVF field.

\section{Validation criterion}

When a ground truth was available (synthetic 4D data, PET image simulations), the segmentation results were compared to the true volumes after convergence by using the Jaccard coefficient $J(A, B)$ that expresses volume similarity [45]. It is the ratio between the intersection and the union of the ground truth volume $(A)$ and the segmented object $(B)$. It ranges from 0 to 1 , with 1 meaning a perfect match:

$$
J(A, B)=\frac{|A \cap B|}{|A \cup B|}
$$

For VGS, only visual results were analyzed, as the high noise levels of the studied images led to multiple spurious surface reconstruction in all the tested images, making the Jaccard score uninterpretable. This issue stressed the need for topology-preserving procedures for such applications, a known drawback of implicit representations [20].

\section{Synthetic 4D BENCHMARK}

We generated 5 synthetic images of dimensions $70 \times 50 \times$ $50 \times 10$ voxels featuring a spherical object of diameter 36 voxels inside a uniform background. In each image, the noisefree background intensity was set to 1 in every channel while the object intensity varied along the channels. By analogy with PET imaging studied hereafter in section VI, the curve that represents the different values of a voxel along the channels 


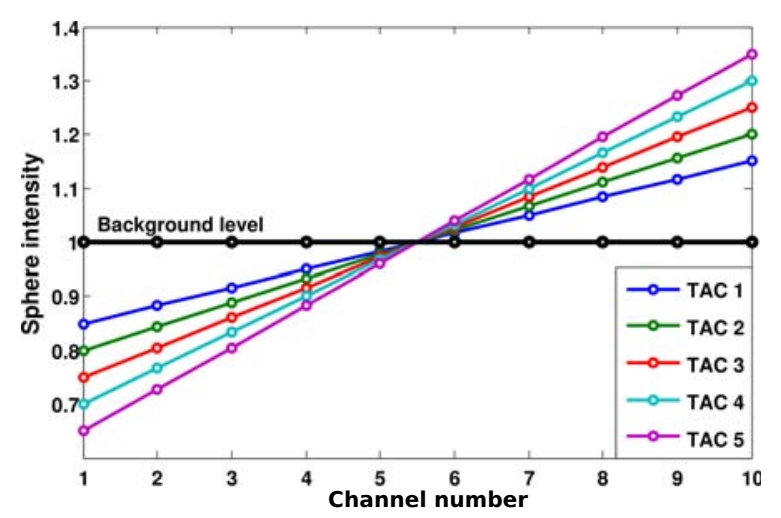

Fig. 4: Intensity values of the sphere object along the 10 channels (TAC) for the 5 images of the synthetic benchmark dataset

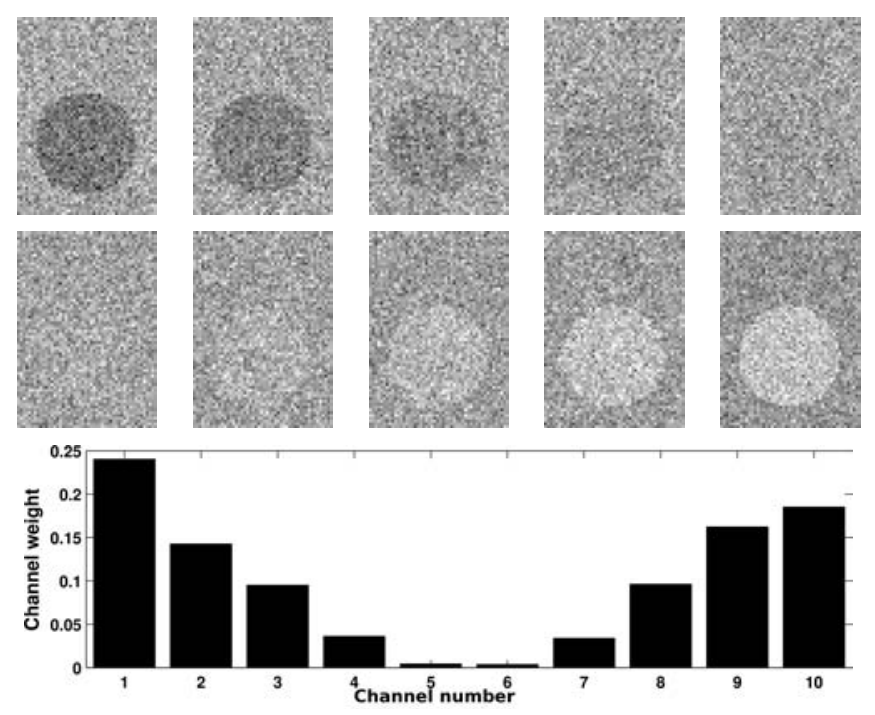

Fig. 5: Top: 2D slices of the 10 channels of a synthetic 4D image that exhibits varying contrast with background. Bottom: corresponding 4DGVF weights obtained for this image

is referred to as its time-activity curve (TAC). We generated 5 4D images for which the object TACs are displayed in Fig. 4. From these noise-free images, a set of noisy 4D images was generated by adding white Gaussian noise in the channels $(\sigma=$ 0.2 ). As a consequence, the number of channels in which the contrast between foreground and background was significantly superior to the noise-induced gradient amplitude varied along the different images of the dataset. These images presented two difficulties that can be found in 4D images, namely low SNR that makes edge detection challenging in individual channels, as well as variations in representativeness of the sphere along the channels.

For the model initialization, a spherical triangulated surface mesh of radius 10 voxels was placed at the center of mass of the object (initial Jaccard index of 0.23).

1) Weighting influence on edge detection: The different channels in a 2D slice of one of the synthetic 4D images are displayed at the top of figure 5. The corresponding

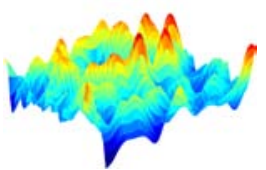

(a)

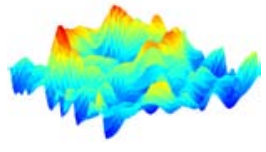

(b)

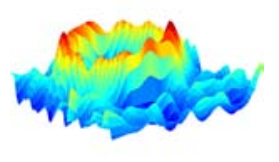

(c)
Fig. 6: Surface plots of the gradient amplitude of a synthetic 4D image: (a) $f_{k}$ (best contrasted channel) (b) $\mathbf{N}_{\mathbb{1}}$ (c) $\mathbf{N}_{\omega}$

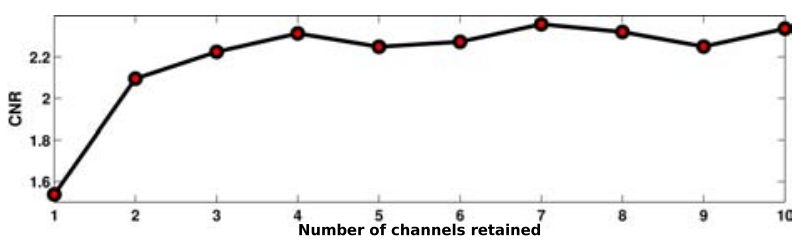

Fig. 7: CNR of vector gradient maps $\mathbf{N}_{\omega}$ averaged over the synthetic benchmark image dataset as a function of the number of high-weight channels used for their calculation

weights obtained with the proposed 4DGVF approach after initialization of the surface model are shown below. In this image, the weighting scheme was consistent with the observed variations of contrast. It was indeed desirable to lower the contribution of channels 4 to 7 in the calculation of the LST.

To study the influence of weighting the LST we compared the proposed weighted magnitude $\mathbf{N}_{\omega}$ to the magnitude $\mathbf{N}_{\mathbb{1}}$ as well as the gradient amplitude $f_{k}$ obtained in the best contrasted channel. Figure 6 displays representative amplitude edge maps of one slice, where the maximum value of each 3D map was set to 1 . The edge signal was enhanced with $\mathbf{N}_{\omega}$ compared to $f_{k}$ and $\mathbf{N}_{\mathbb{1}}$, and in homogeneous regions, spurious variations due to noise were kept at lower levels, leading to better edge detection with the $\mathbf{N}_{\omega}$ amplitude edge map.

We studied quantitatively the quality of the vector gradient amplitude $\mathbf{N}_{\omega}$ as a function of the number of high contrast channels used for its calculation. We computed $\mathbf{N}_{\omega}$ using varying numbers of high contrast channels, ranging from 1 (best channel only) to 10 (all channels included: proposed method). The criterion for assessing the quality of the resulting amplitude maps was the CNR of $\mathbf{N}_{\omega}$ :

$$
C N R=\left|\mu_{e}-\mu_{b g}\right| / \sigma_{b g},
$$

where $\mu_{e}$ is the average intensity value of edge voxels, $\mu_{b g}$ is the average intensity of non-edge voxels (background), and $\sigma_{b g}$ is the standard deviation of the background. Fig. 7 displays CNR for $\mathbf{N}_{\omega}$ maps averaged over the dataset as a function of the number of channels (ordered by decreasing contrast) used for their calculation. On average, using the $n=7$ channels with the highest contrast values led to maximum CNR for $\mathbf{N}_{\omega}(\mathrm{CNR}=2.36)$, with an increase of about $53 \%$ compared with the CNR obtained by only using the highest contrasted channel $(n=1, \mathrm{CNR}=1.53)$. Including all channels with the proposed weighted averaging did not lower significantly the CNR of the edge maps compared to the maximum value $(n=10, \mathrm{CNR}=2.34)$. This is due to the fact that low contrast 


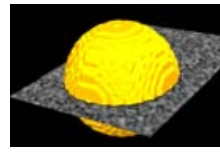

(a)

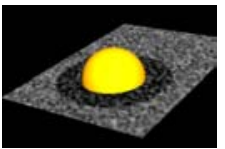

(b)

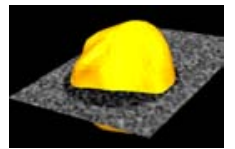

(c)

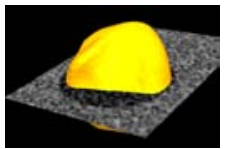

(d)

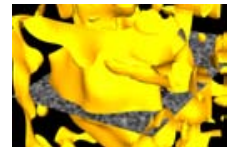

(e)

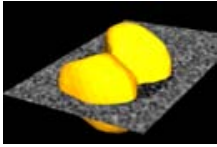

(f)

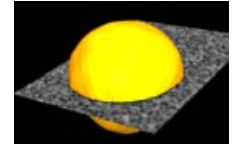

(g)

Fig. 8: Representative segmentation results for an image of the synthetic $4 \mathrm{D}$ benchmark image set. (a) ground truth (b) initial shape (c) best GGVF (d) best VFC (e) VGS (f) CGVF (g) 4DGVF

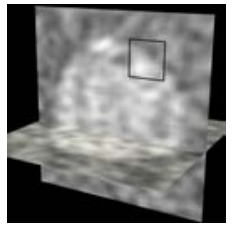

(a)

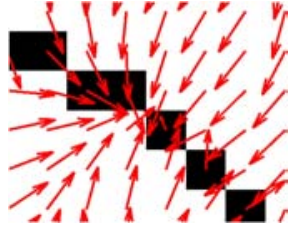

(b)

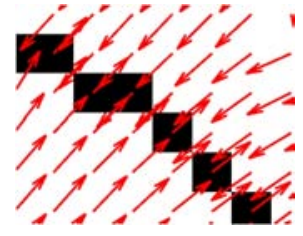

(c)

Fig. 9: (a) Representative channel of a synthetic 4D image where the region that was analyzed here is outlined, (b) CGVF field based on $\mathbf{N}_{\omega}$ and $\nabla \mathbf{N}_{\omega}$, (c) $4 \mathrm{DGVF}$ field based on both $\mathbf{N}_{\omega}$ and $\overrightarrow{\mathbf{V}}$

channels have a reduced influence on the LST calculation with the proposed blind weighting scheme. In addition, this strategy does not require any prior selection of the number of channels that are considered valuable for edge localization.

2) Orientations of vector edges: We evaluated the accuracy of the force field around edges by comparing the 4DGVF field based on the diffusion of $\overrightarrow{\mathbf{V}}$ to a CGVF field based on the diffusion of $\nabla \mathbf{N}_{\omega}$ rather than $\nabla \mathbf{N}_{\mathbb{1}}$, in order to remove the influence of the weighting scheme.

Figure 9 shows a typical behaviour of these two fields in a noisy 4D image of the synthetic dataset. Near the contour, some CGVF vectors collapsed around an edge voxel (Fig. 9b). On the contrary, the 4DGVF field was oriented toward the contour (Fig. 9c). This example illustrates the advantage of constraining the directions of the 4DGVF field acccording to the principal eigenvector of the structure tensor $G_{\omega}$ around edges.

3) Segmentation performances: Figure 8 displays segmentation results on an image of the synthetic dataset for the tested approaches. In this image, the 4DGVF model was able to recover the sphere shape, while the other approaches partially failed due to excessive noise levels. Single-channel approaches (e.g. Fig. 8b and Fig. 8c) obtained comparatively worse segmentation results due to the fact that all channels, including the best-contrasted ones, were too corrupted by noise for unambiguous edge detection. The color GVF snake, although benefiting from vector-valued data, did not capture the sphere shape entirely (Fig. 8e). The vector geometric snake was even more penalized due to its topological flexibility, leading to arbitrary surface reconstructions throughout the image (Fig. 8d). For all tested images, the 4DGVF method visually outperformed the other approaches.

Table II shows quantitative results averaged over the synthetic benchmark image dataset. For each image, a single result was obtained for vector-valued models (CGVF and 4DGVF)

while we retained the channel corresponding to the best Jaccard similarity coefficient for single-channel approaches (GGVF and VFC). Overall, analysis of the Jaccard results suggests that the 4DGVF model improved segmentation results. CGVF provided the second best results after 4DGVF on average, but single channel approaches were able to perform better than CGVF in sufficiently well contrasted channels. The 4DGVF scheme was less hampered by the low contrast channels than CGVF due to the weighting scheme.

TABLE II: Jaccard scores for the synthetic 4D benchmark image set

\begin{tabular}{ccccc}
\hline \hline Initial & $\begin{array}{c}\text { GGVF } \\
\text { (best channel) }\end{array}$ & $\begin{array}{c}\text { VFC } \\
\text { (best channel) }\end{array}$ & CGVF & 4DGVF \\
\hline 0.23 & $0.79 \pm 0.13$ & $0.76 \pm 0.13$ & $0.74 \pm 0.23$ & $\mathbf{0 . 8 6} \pm \mathbf{0 . 1 2}$ \\
\hline \hline
\end{tabular}

\section{VALIDATION ON DYNAMIC PET IMAGES}

Dynamic PET imaging consists in the successive acquisition of different time frames of an identical field of view. A dynamic PET image can therefore be considered as a vector-valued image, where each time frame of the sequence corresponds to one channel. The resulting images reflect the dynamics of a radiotracer concentration in the body, but suffer from low resolution and low SNR. In these images, regions of interest, or kinetic regions have varying contrasts with respect to the surrounding regions over time. PET imaging is a functional imaging modality that can provide information unavailable in structural imaging modalities such as computed tomography. There has been a growing interest for its application to the early diagnosis of neurodegenerative pathologies such as Alzheimer's disease [46], [47] or amyotrophic lateral sclerosis [48], and in the study of neuroinflammation [49], [50].

The validation of segmentation results using real clinical images is difficult due to lack of ground truth. While we show examples of application to real data in section VI-E, we assess the 4DGVF approach with quantitative results on realistic Monte Carlo simulations of dynamic PET images.

\section{A. PET-SORTEO benchmark image database}

We used simulations of $\left[{ }^{11} \mathrm{C}\right]$-Raclopride dynamic $3 \mathrm{D}+\mathrm{t}$ PET images of the brain from the publicly available PETSORTEO benchmark image database (http://sorteo.cermep.fr) [51]. We focused on the segmentation of the putamen in both cerebral hemispheres. These realistic images account for the inter-individual variability of anatomical structures by using 


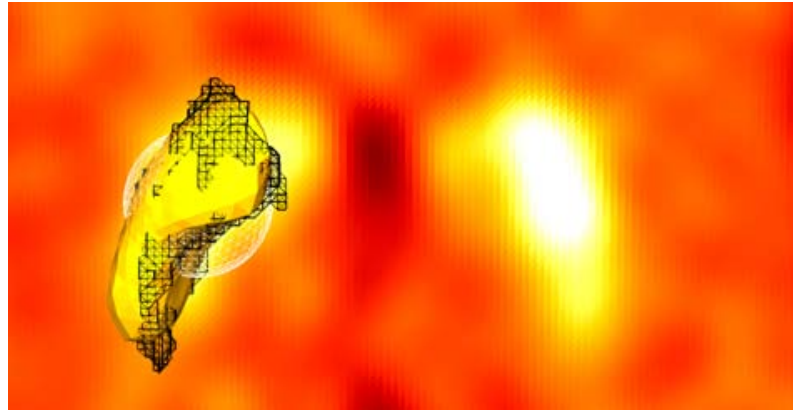

Fig. 10: Segmentation of the left putamen superimposed with a transaxial slice of image P02 of the PET-SORTEO benchmark. Ground truth (black wireframe), initial model (white wireframe) and 4DGVF result after convergence (solid yellow)

TABLE III: Jaccard segmentation results for the left (L) and right $(\mathrm{R})$ putamina in the PET-SORTEO benchmark image dataset

\begin{tabular}{cccccccccc}
\hline \hline Method & \multicolumn{2}{c}{ GGVF } & \multicolumn{2}{c}{ VFC } & \multicolumn{2}{c}{ CGVF } & \multicolumn{2}{c}{ 4DGVF } \\
& \multicolumn{2}{c}{ (best ch.) } & \multicolumn{2}{c}{ (best ch.) } & & & & \\
& L & R & L & R & L & R & L & R \\
\hline Image & & & & & & & & \\
Jacob & 0.65 & 0.68 & 0.67 & 0.68 & 0.62 & 0.63 & $\mathbf{0 . 7 7}$ & $\mathbf{0 . 7 7}$ \\
P02 & 0.61 & 0.58 & 0.62 & 0.61 & 0.60 & 0.61 & $\mathbf{0 . 6 8}$ & $\mathbf{0 . 7 0}$ \\
P03 & 0.61 & 0.58 & 0.62 & 0.61 & 0.60 & 0.54 & $\mathbf{0 . 6 5}$ & $\mathbf{0 . 6 6}$ \\
P04 & 0.57 & 0.58 & 0.59 & 0.61 & 0.48 & 0.58 & $\mathbf{0 . 6 0}$ & $\mathbf{0 . 6 4}$ \\
\hline \hline
\end{tabular}

different real MR images as numerical head models. Each dynamic PET volume has dimensions of $128 \times 128 \times 63 \times 26$ voxels, while each MR volume contains $181 \times 217 \times 181$ voxels. We limited our experiments to 4 images of the benchmark: the Jacob, P02, P03 and P04 images. Each of the dynamic PET images were registered to their corresponding MR volumes with a rigid registration algorithm using the medical imaging software PMOD v.3.4. The provided labeled MR images were used as ground truth for the validation of the segmentation results. For a fair comparison, we used for this dataset identical initial ellipsoid models for every method. We centered a sphere at the center of mass of the considered putamen in the corresponding labeled MR image. The radius of the sphere was set to 10 voxels (initial Jaccard index of 0.46 on average).

Figure 10 shows a cropped transaxial slice in the $20^{\text {th }}$ channel of the P02 image around the putamina (slice 69/181). The 3D surfaces of the ground truth, of the initial model, and of the 4DGVF model after convergence are showed in black wireframe, white wireframe and solid yellow respectively. The 4DGVF model was able to capture the shape of the left putamen. For this image, the Jaccard score of the 4DGVF model after convergence was 0.68 . According to this criteria, the 4DGVF model outperformed the other approaches for both left and right putamen segmentation on all tested images (table III).

\section{B. Simulations of realistic $4 D$ PET images with GATE}

Additional dynamic PET images were simulated using GATE, a highly realistic medical image simulator based on

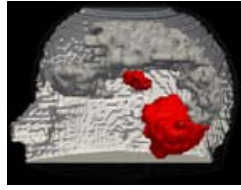

(a)

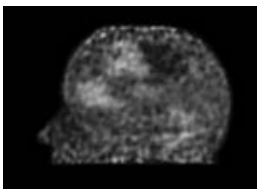

(b)

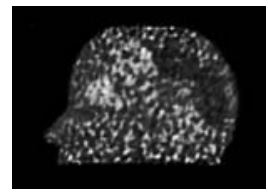

(c)
Fig. 11: Simulations of dynamic PET images. (a) Zubal phantom (b) Zubal simulation (mid SNR) (c) Zubal simulation (low SNR)
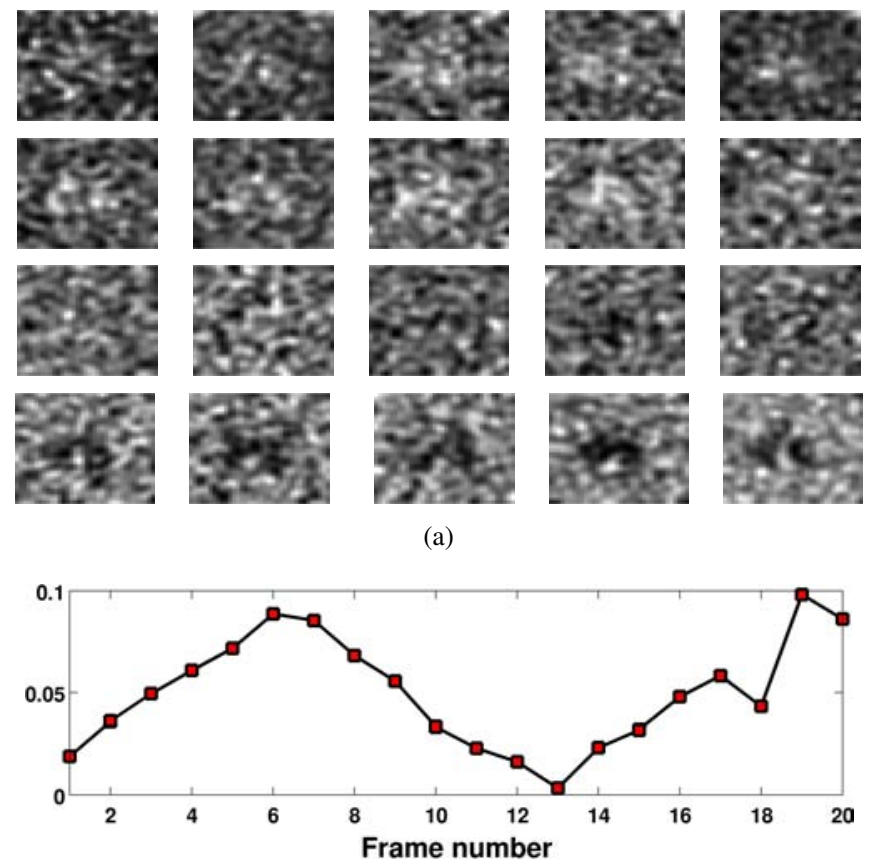

(b)
Fig. 12: (a) 2D transverse slices of the 20 frames of the low SNR Zubal simulation around the thalamus. (b) 4DGVF weights per frame for simulation shown in (a)

the CERN's GEANT4 particle interaction platform [52], [53].

We used the Zubal head phantom [54], describing the main brain structures as a voxelized source. Six regions were considered for the simulation: cerebellum, thalamus, parietal, frontal and occipital lobes, and the remaining parts of the brain were the background (Fig. 11a).

Time-activity curves, which represent the variations of each voxel intensity along the time frames, were generated according to a three-compartment model [55] that models the kinetics of the radiotracer in the body. The reconstruction of the PET image was performed using a fully 3D OP-OSEM (ordinary Poisson ordered-subset expectation-maximization) iterative method into $2.2 \times 2.2 \times 2.8 \mathrm{~mm}^{3}$ voxels. We performed two different reconstructions: one using 2 iterations and 16 subsets (figure 11b) and one using 10 iterations and 16 subsets (figure 11c) that resulted in different levels of SNR, called mid and low respectively. The simulation of these two images required 90 days of parallel computations on a 12 cores 48 GB RAM computer. 


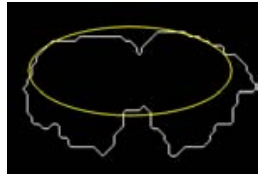

(a)

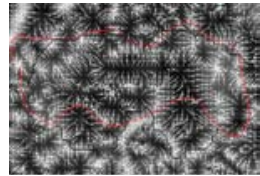

(b)

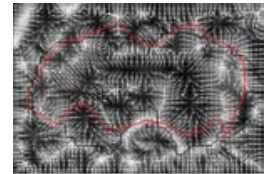

(c)

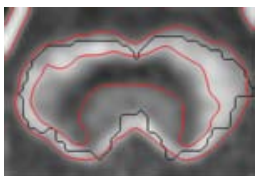

(d)

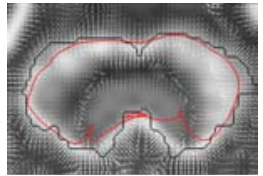

(e)

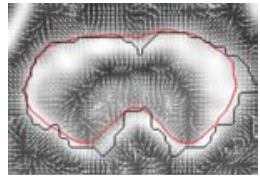

(f)

Fig. 13: Segmentation results for cerebellum in the mid SNR Zubal simulation illustrated on a selected slice. Solid lines represent intersections of mesh with slice. The black contour represents the ground truth surface. (a) Ground truth (white), initial shape (yellow) (b) GGVF in one frame (c) VFC in the same frame (d) VGS (e) CGVF (f) 4DGVF

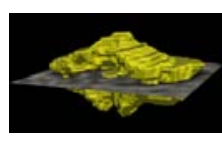

(a)

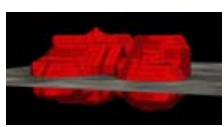

(h)

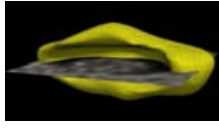

(b)

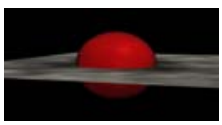

(i)

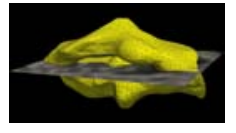

(c)

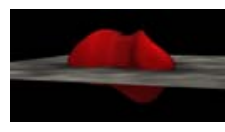

(j)

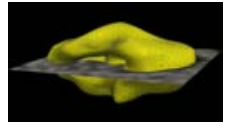

(d)

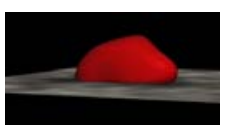

(k)

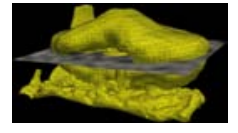

(e)

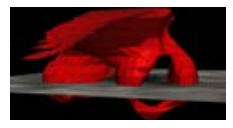

(1)

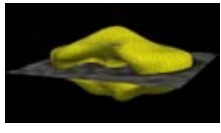

(f)

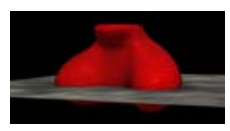

(m)

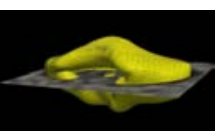

(g)

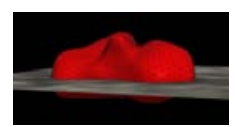

(n)

Fig. 14: Representative segmentation results in the low SNR Zubal simulation. First row: cerebellum. Scond row: thalamus. $(a, h)$ ground truth. (b,i) initial shape. $(c, j)$ GGVF in the best frame $(d, k)$ VFC in the best frame (e,l) VGS (f,m) CGVF (g,n) Proposed 4DGVF

For each of the reconstructed images of the Zubal head phantom, we studied the segmentation of the cerebellum and of the thalamus, two structures showing different kinetics and volumes, colored in red in figure $11 \mathrm{a}$.

\section{Weighting scheme}

Figure 12a displays 2D transverse slices of the 20 frames of the low SNR Zubal simulation around the thalamus. Thalamus can be distinguished as a hypersignal in frames of the second row and as a hyposignal in late frames. Estimated weights for the calculation of the corresponding LST are shown in figure $12 \mathrm{~b}$ after convergence of the model. The 4DGVF weights were in good agreement with the subjective quality observed in each channel: the weight values followed the variations of contrast along the channels and thereby the representativeness of the object.

\section{Segmentation performances on $4 D$ PET simulations}

Figure 13 illustrates segmentation results in the mid SNR Zubal simulation around the cerebellum. 2D slices of the results are displayed for the sake of readability. The first column shows the ground truth and the ellipsoidal shape used as initialization. Columns b-f present segmentation results of the tested methods superimposed with the edge map used by the method and with the corresponding external force field. The intersection of the active surface with the slice is depicted as a red line and the ground truth as a black line. While GVF, VFC, CGVF and 4DGVF all led to consistent cerebellum segmentation, the best result was achieved with 4DGVF, followed by CGVF. 4DGVF was able to better capture the concavity of the cerebellum formed by the fourth ventricle (bottom part of figure 13f). Again, the implicit VGS model created numerous splitted reconstructed surfaces of various sizes and shapes and hence was not quantitatively evaluated.

Figure 14 shows 3D representative segmentation results in the low SNR simulation for all tested methods, either using the VPIG initialization (top row, cerebellum) or using an ellipsoidal initialization (bottom row, thalamus). For both initialization methods, the overall shape of the two objects was best recovered by the 4DGVF approach. Despite low SNR conditions, the 4DGVF approach was able to capture the thalamus, a small region compared to the voxel size. The second best segmentation of the thalamus was obtained with CGVF, with segmentation results that were however visually less precise than the 4DGVF model (Fig. 14f and Fig. 14m).

Table IV shows the quantitative similarity criteria between the segmentation results and the ground truth after convergence for the two different simulations. For both images, segmentation results were improved by the 4DGVF approach. In the case of the segmentation of the cerebellum, the best VFC and GGVF results produced relatively high Jaccard. However, single-channels approaches require to select the channels of interest a priori, which is not always feasible in practice. On the contrary, a single segmentation result was obtained for vector-valued models, with systematic improvement observed for 4DGVF.

In general, the 4DGVF external force field benefited from comparatively larger attraction range than other approaches, allowing initialization from farther distances, which is confirmed by the stability of the segmentation result against the initialization. The cumulative effect of exploiting accurate gradient directions drawn from the vectorial edge map and of weighting the image channels led to better performance 
of 4DGVF over CGVF, which obtained second best results on average. The improved robustness of $4 \mathrm{DGVF}$ under low SNR is emphasized in the case of the noisier Zubal simulation, where the proposed method led to distinct improvements of figures of merit.

TABLE IV: Segmentation results for the Zubal simulations

\begin{tabular}{|c|c|c|c|c|}
\hline \multirow[b]{2}{*}{ Initialization } & \multicolumn{4}{|c|}{ Jaccard index } \\
\hline & Ellipsoid & VPIG & Ellipsoid & VPIG \\
\hline \multicolumn{5}{|l|}{ Zubal mid SNR } \\
\hline & \multicolumn{2}{|c|}{ Thalamus } & \multicolumn{2}{|c|}{ Cerebellum } \\
\hline Initial score & 0.23 & 0.41 & 0.48 & 0.65 \\
\hline GGVF (best channel) & 0.36 & 0.47 & 0.82 & 0.83 \\
\hline VFC (best channel) & 0.42 & 0.57 & 0.82 & 0.82 \\
\hline CGVF & 0.56 & 0.56 & 0.82 & 0.81 \\
\hline 4DGVF & 0.61 & 0.64 & 0.83 & 0.85 \\
\hline \multicolumn{5}{|l|}{ Zubal low SNR } \\
\hline & \multicolumn{2}{|c|}{ Thalamus } & \multicolumn{2}{|c|}{ Cerebellum } \\
\hline Initial score & 0.23 & 0.36 & 0.48 & 0.61 \\
\hline GGVF (best channel) & 0.36 & 0.48 & 0.72 & 0.77 \\
\hline VFC (best channel) & 0.45 & 0.46 & 0.75 & 0.79 \\
\hline CGVF & 0.49 & 0.50 & 0.64 & 0.81 \\
\hline 4DGVF & 0.55 & 0.55 & 0.79 & 0.84 \\
\hline
\end{tabular}

In the tested images, the weighting scheme led to systematic enhancement of the gradient magnitude map $\mathbf{N}_{\omega}$ over $\mathbf{N}_{\mathbb{1}}$ to the benefit of the 4DGVF approach. The most computationally expensive aspect of the method lies in the re-calculation of the force field due to the re-evaluation of the weights at each iteration. However, in practice, recalculating weights at each iteration is not necessary because they converge to a steady result along with the surface model. For example, one can re-evaluate weights depending on the amount of global deformation of the model, as small deformations are likely to cause negligible change in the weights. While the proposed weighting scheme is convenient for numerous modalities and applications, the 4DGVF approach can be enriched by other type of weights, also based for example on noise estimation or available a priori knowledge.

\section{E. Illustration on real data}
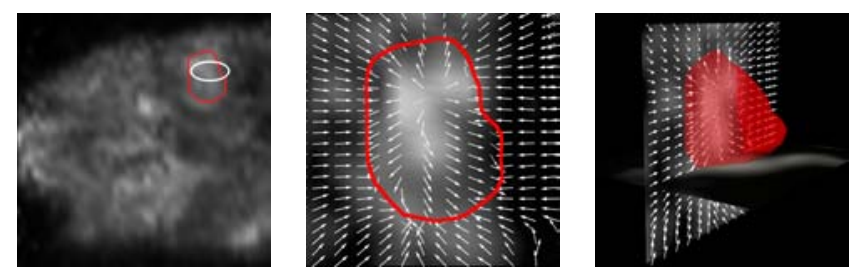

Fig. 15: Segmentation of a quinolinic acid lesion in the striatum region of a rat brain. Result is shown in red, initial shape in white (left), superimposed onto the 4DGVF field (middle, right)

To illustrate the behavior of the $4 \mathrm{DGVF}$ approach in a pre clinical context, we performed a dynamic PET acquisition of a rat using [18F]-DPA-714 injections, a radiotracer specific to the translocator protein (TSPO). This protein is over expressed under pathologic neuroinflammatory conditions and can therefore measure active diseases in the brain. The inflammation was produced by performing unilateral quinolinic acid lesions in the right striatum of the rat. Images were acquired on a microPET-CT GE Vista in list-mode and reconstructed using 3D-OSEM iterative method with corrections for attenuation, random and scattered coincidences. 27 time frames of $175 \times 175 \times 61$ voxels of $0.39 \times 0.39 \times 0.78 \mathrm{~mm}^{3}$ were reconstructed over a period of 50 minutes according to the following protocol: $4 \times 10$ s, $4 \times 20$ s, $6 \times 60$ s, $10 \times 80$ s, $3 \times 600$ s.

Figure 15 shows a representative segmentation result for one rat. The shape produced was consistent with the morphology and location of the injured region.

\section{CONCLUSION}

We have proposed a novel external force field for the segmentation of vector-valued images using parametric active surfaces. The proposed 4DGVF field enables the segmentation of noisy $4 \mathrm{D}$ images where edge information cannot be deduced from a single channel, and where only the redundancy of edge information along the channels can help recover the feature of interest. In the 4DGVF approach, the gradient signal is weighted according to a blind estimation of contrast, favouring channels in which edges are better defined. While existing approaches based on deformable models applied to vectorvalued images exploit local structure information in a scalar way to define vector edges, the $4 \mathrm{DGVF}$ field is produced through nonlinear diffusion of a vectorial edge map computed from the eigenvector analysis of the local structure tensor, improving robustness to noise.

Quantitative assessment on synthetic images and realistic simulations, and results on real dynamic PET images confirmed the potential impact of the method for segmentation of vector-based 2D or 3D imaging modality, such as dynamic PET, functional MRI or hyper-spectral imaging.

\section{ACKNOWLEDGMENT}

The research leading to these results has received funding from the European Union's Seventh Framework Programme (FP7/2007-2013) under grant agreement HEALTH-F2-2011278850 (INMiND).

\section{REFERENCES}

[1] T.S. Lee, D. Mumford, and A. Yuille, "Texture segmentation by minimizing vector-valued energy functionals: The coupled-membrane model," in Computer Vision ECCV'92. Springer, 1992, pp. 165-173.

[2] G. Sapiro, "Vector (self) snakes: A geometric framework for color, texture, and multiscale image segmentation," in Image Processing, 1996. Proceedings., International Conference on. IEEE, 1996, vol. 1, pp. 817820.

[3] T.F. Chan, B.Y. Sandberg, and L. Vese, "Active contours without edges for vector-valued images," Journal of Visual Communication and Image Representation, vol. 11, no. 2, pp. 130-141, 2000.

[4] M. Rousson and R. Deriche, "A variational framework for active and adaptative segmentation of vector valued images," in Motion and Video Computing, 2002. Proceedings. Workshop on. IEEE, 2002, pp. 56-61.

[5] D. Tschumperle and R. Deriche, "Vector-valued image regularization with pdes: A common framework for different applications," Pattern Analysis and Machine Intelligence, IEEE Transactions on, vol. 27, no. 4, pp. 506-517, 2005.

[6] M. Kass, A. Witkin, and D. Terzopoulos, "Snakes: Active contour models," International journal of computer vision, vol. 1, no. 4, pp. 321-331, 1988. 
[7] T. McInerney and D. Terzopoulos, "Deformable models in medical image analysis," in Mathematical Methods in Biomedical Image Analysis, 1996., Proceedings of the Workshop on. IEEE, 1996, pp. 171-180.

[8] C. Xu and J.L. Prince, "Snakes, shapes, and gradient vector flow," Image Processing, IEEE Transactions on, vol. 7, no. 3, pp. 359-369, 1998.

[9] C. Xu and J.L. Prince, "Generalized gradient vector flow external forces for active contours," Signal Processing, vol. 71, no. 2, pp. 131-139, 1998.

[10] B. Li and S.T. Acton, "Active contour external force using vector field convolution for image segmentation," Image Processing, IEEE Transactions on, vol. 16, no. 8, pp. 2096-2106, 2007.

[11] T.F. Chan and L. Vese, "Active contours without edges," Image Processing, IEEE Transactions on, vol. 10, no. 2, pp. 266-277, 2001.

[12] N. Paragios and R. Deriche, "Geodesic active regions: A new framework to deal with frame partition problems in computer vision," Journal of Visual Communication and Image Representation, vol. 13, no. 1, pp. 249-268, 2002.

[13] X. Xie and M. Mirmehdi, "Rags: Region-aided geometric snake," Image Processing, IEEE Transactions on, vol. 13, no. 5, pp. 640-652, 2004.

[14] S. Lankton and A. Tannenbaum, "Localizing region-based active contours," Image Processing, IEEE Transactions on, vol. 17, no. 11, pp. 2029-2039, 2008

[15] D. Barbosa, T. Dietenbeck, J. Schaerer, J. D'hooge, D. Friboulet, and O. Bernard, "B-spline explicit active surfaces: An efficient framework for real-time 3-d region-based segmentation," Image Processing, IEEE Transactions on, vol. 21, no. 1, pp. 241-251, 2012.

[16] X. Xie and M. Mirmehdi, "Mac: Magnetostatic active contour model," Pattern Analysis and Machine Intelligence, IEEE Transactions on, vol. 30, no. 4, pp. 632-646, 2008

[17] S. Kichenassamy, A. Kumar, P. Olver, and A. Tannenbaum, A.and Yezzi, "Gradient flows and geometric active contour models," in Computer Vision, 1995. Proceedings., Fifth International Conference on. IEEE, 1995, pp. 810-815.

[18] V. Caselles, R. Kimmel, and G. Sapiro, "Geodesic active contours," International journal of computer vision, vol. 22, no. 1, pp. 61-79, 1997.

[19] M. Rousson and N. Paragios, "Shape priors for level set representations," in Computer Vision ECCV 2002, pp. 78-92. Springer, 2002.

[20] C. Xu X. Han and J.L. Prince, "A topology preserving level set method for geometric deformable models," Pattern Analysis and Machine Intelligence, IEEE Transactions on, vol. 25, no. 6, pp. 755-768, 2003.

[21] K.W. Sum and P. Cheung, "Boundary vector field for parametric active contours," Pattern Recognition, vol. 40, no. 6, pp. 1635-1645, 2007.

[22] P. Ghosh, L. Bertelli, B. Sumengen, and B.S. Manjunath, "A nonconservative flow field for robust variational image segmentation," Image Processing, IEEE Transactions on, vol. 19, no. 2, pp. 478-490, 2010.

[23] D. Zeng, Z. Zhou, and S. Xie, "Image segmentation based on the poincaré map method," Image Processing, IEEE Transactions on, vol. 21, no. 3, pp. 946-957, 2012

[24] J. Canny, "A computational approach to edge detection," Pattern Analysis and Machine Intelligence, IEEE Transactions on, , no. 6, pp. 679-698, 1986.

[25] C. Tauber, H. Batatia, and A. Ayache, "Quasi-automatic initialization for parametric active contours," Pattern Recognition Letters, vol. 31, no. 1, pp. 83-90, 2010.

[26] B. Li and S.T. Acton, "Automatic active model initialization via poisson inverse gradient," Image Processing, IEEE Transactions on, vol. 17, no. 8, pp. 1406-1420, 2008.

[27] J. Cheng and S.W. Foo, "Dynamic directional gradient vector flow for snakes," Image Processing, IEEE Transactions on, vol. 15, no. 6, pp. 1563-1571, 2006.

[28] G. Sapiro, "Color snakes," Computer Vision and Image Understanding, vol. 68, no. 2, pp. 247-253, 1997.

[29] R. Goldenberg, R. Kimmel, E. Rivlin, and M. Rudzsky, "Fast geodesic active contours," Image Processing, IEEE Transactions on, vol. 10, no. 10, pp. 1467-1475, 2001

[30] L. Yang, P. Meer, and D.J. Foran, "Unsupervised segmentation based on robust estimation and color active contour models," Information Technology in Biomedicine, IEEE Transactions on, vol. 9, no. 3, pp. 475-486, 2005.

[31] S. Di Zenzo, "A note on the gradient of a multi-image," Computer Vision, Graphics, and Image Processing, vol. 33, no. 1, pp. 116-125, 1986.

[32] T. Brox, From pixels to regions: partial differential equations in image analysis, Ph.D. thesis, 2005.

[33] G. Sapiro and D.L. Ringach, "Anisotropic diffusion of multivalued images with applications to color filtering," Image Processing, IEEE Transactions on, vol. 5, no. 11, pp. 1582-1586, 1996.
[34] J. Weickert, "Coherence-enhancing diffusion of colour images," Image and Vision Computing, vol. 17, no. 3, pp. 201-212, 1999.

[35] J. Zhang, J. Zheng, and J. Cai, "A diffusion approach to seeded image segmentation," in Computer Vision and Pattern Recognition (CVPR), 2010 IEEE Conference on. IEEE, 2010, pp. 2125-2132.

[36] L.D. Cohen and I. Cohen, "Finite-element methods for active contour models and balloons for 2-d and 3-d images," Pattern Analysis and Machine Intelligence, IEEE Transactions on, vol. 15, no. 11, pp. 11311147, 1993.

[37] A. Cumani, "Edge detection in multispectral images," CVGIP: Graphical models and image processing, vol. 53, no. 1, pp. 40-51, 1991.

[38] J. Weickert, "Coherence-enhancing diffusion filtering," International Journal of Computer Vision, vol. 31, no. 2-3, pp. 111-127, 1999.

[39] G. Piella, "Image fusion for enhanced visualization: A variational approach," International Journal of Computer Vision, vol. 83, no. 1, pp. 1-11, 2009.

[40] G. VanAndel, H.W. Venema, C.B. Majoie, G.J. Den Heeten, C.A. Grimbergen, and G.J. Streekstra, "Intracranial ct angiography obtained from a cerebral ct perfusion examination," Medical physics, vol. 36, no. 4, pp. 1074-1085, 2009.

[41] P. Blomgren and T.F. Chan, "Color tv: total variation methods for restoration of vector-valued images," Image Processing, IEEE Transactions on, vol. 7, no. 3, pp. 304-309, 1998.

[42] W.E. Lorensen and H.E. Cline, "Marching cubes: A high resolution 3d surface construction algorithm," in ACM Siggraph Computer Graphics. ACM, 1987, vol. 21, pp. 163-169.

[43] G. Thürrner and C. A. Wüthrich, "Computing vertex normals from polygonal facets," Journal of Graphics Tools, vol. 3, no. 1, pp. 43-46, 1998.

[44] G. Taubin, "A signal processing approach to fair surface design," in Proceedings of the 22nd annual conference on Computer graphics and interactive techniques. ACM, 1995, pp. 351-358.

[45] P. Jaccard, "Distribution de la flore alpine dans le bassin des Dranses et dans quelques régions voisines," Bulletin de la Société Vaudoise des Sciences Naturelles, vol. 37, pp. 241-272, 1901.

[46] V. Camus, P. Payoux, L. Barré, B. Desgranges, T. Voisin, C. Tauber, R. La Joie, M. Tafani, C. Hommet, G. Chételat, et al., "Using pet with 18f-av-45 (florbetapir) to quantify brain amyloid load in a clinical environment," European journal of nuclear medicine and molecular imaging, vol. 39, no. 4, pp. 621-631, 2012.

[47] C. Tauber, E. Beaufils, C. Hommet, M. J. Ribeiro, J. Vercouillie, E. Vierron, K. Mondon, J.-P. Cottier, V. Gissot, D. Guilloteau, et al., "Brain [18 f] fddnp binding and glucose metabolism in advanced elderly healthy subjects and alzheimer's disease patients," Journal of Alzheimer's Disease, 2013.

[48] P. Corcia, C. Tauber, J. Vercoullie, N. Arlicot, C. Prunier, J. Praline, G. Nicolas, Y. Venel, C. Hommet, J.-L. Baulieu, et al., "Molecular imaging of microglial activation in amyotrophic lateral sclerosis," PloS one, vol. 7, no. 12, pp. e52941, 2012.

[49] F. E. Turkheimer, P. Edison, N. Pavese, F. Roncaroli, A. N. Anderson, A. Hammers, A. Gerhard, R. Hinz, Y.F. Tai, and D. J. Brooks, "Reference and target region modeling of [11c]-(r)-pk11195 brain studies," Journal of Nuclear Medicine, vol. 48, no. 1, pp. 158-167, 2007.

[50] M. Yaqub, B.N.M. van Berckel, A. Schuitemaker, R. Hinz, F.E. Turkheimer, G. Tomasi, A.A. Lammertsma, and R. Boellaard, "Optimization of supervised cluster analysis for extracting reference tissue input curves in (r)-\&lsqb; 11c\&rsqb; pk11195 brain pet studies," Journal of Cerebral Blood Flow \& Metabolism, vol. 32, no. 8, pp. 1600-1608, 2012.

[51] A. Reilhac, G. Batan, C. Michel, C. Grova, J. Tohka, D. Collins, Nicolas Costes, and A. Evans, "Pet-sorteo: validation and development of database of simulated pet volumes," Nuclear Science, IEEE Transactions on, vol. 52, no. 5, pp. 1321-1328, 2005.

[52] S. Jan, G. Santin, D. Strul, S. Staelens, K. Assie, D. Autret, S. Avner, R. Barbier, M. Bardies, and PM et al. Bloomfield, "Gate: a simulation toolkit for pet and spect," Physics in medicine and biology, vol. 49, no. 19, pp. 4543, 2004.

[53] S Jan, D Benoit, E Becheva, T Carlier, F Cassol, P Descourt, T Frisson, L Grevillot, L Guigues, L Maigne, et al., "Gate v6: a major enhancement of the gate simulation platform enabling modelling of ct and radiotherapy," Physics in medicine and biology, vol. 56, no. 4, pp. 881, 2011.

[54] G. Zubal, C.R. Harrell, E.O. Smith, Z. Rattner, G. Gindi, P.B. Hoffer, et al., "Computerized three-dimensional segmented human anatomy," Medical Physics-New York-Institute of Physics, vol. 21, no. 2, pp. 299302, 1994. 
[55] M. Kamasak, C. Bouman, E. Morris, and K. Sauer, "Direct reconstruction of kinetic parameter images from dynamic pet data," Medical Imaging, IEEE Transactions on, vol. 24, no. 5, pp. 636-650, 2005.

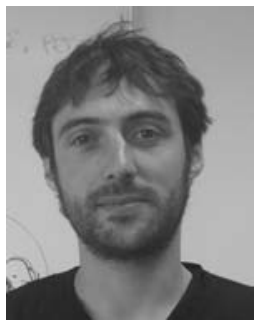

Vincent Jaouen has received the Master degree in applied physics from the University of Rennes 1, France in 2012. Since then, he is pursuing the Ph.D. degree at the INSERM-François Rabelais University unit "Imaging and Brain", Tours, France. He is also a teaching assistant in signal processing at the INSA (National Institute of Applied Sciences) school of engineering, Blois, France. His main research interests are PDE-based segmentation and filtering approaches applied to medical image analysis. He is interested in particular in quantifying neuroinflammatory disorders with PET imaging.

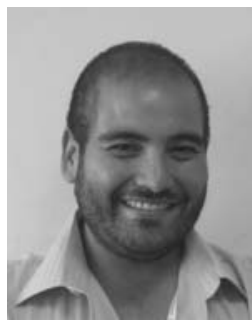

Paulo González is a computer engineer from Universidad Católica del Maule, Talca, Chile. He has received the M.Sc. degree in Computer Sciences from Universidad de Concepción, Chile in 2009. He is currently pursuing the Ph.D. degree at the unit "Imaging and Brain", Tours, France. Since 2009 he is a lecturer and researcher at Universidad Catolica del Maule. His main research interests are related to PDE-based processes applied to PET images, enhancement algorithms and mathematical morphology.

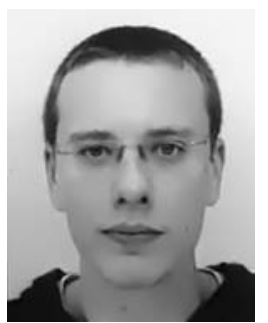

Simon Stute is a researcher at the Service Hospitalier Frédéric Joliot for the Nuclear Energy Agency (CEA) in France. He received his $\mathrm{PhD}$ in 2010, regarding his work on the development and use of realistic Monte Carlo simulations for improved quantification in PET. He is now part of the Biomedical Physics team, and is specialized in the development and application of new image reconstruction techniques in PET.

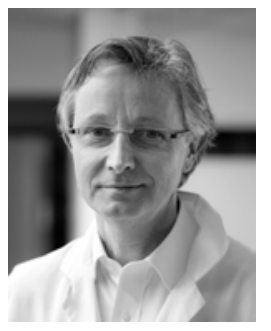

Denis Guilloteau , pharmacist, biologist, is involved in routine in hospital as the head of Department of Nuclear Medicine in the CHRU Bretonneau in Tours and leads researches in the field of Molecular Imaging and more particularly in the development of new radiopharmaceuticals. Professor Denis Guilloteau manages the "Imaging and Brain" INSERM unit that includes radiochemist, mathematicians, biologists, radiologist, neurologists, geriatricians and psychiatrists. One of the objectives of this unit is to develop imaging techniques applicable to the Alzheimer's disease for the diagnosis and the follow-up of the efficiency of treatments. He has published more than 160 international articles.

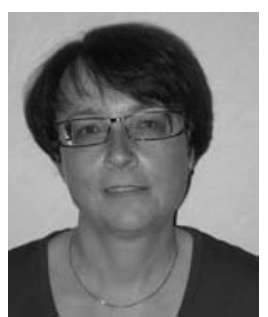

Sylvie Chalon neurobiologist, is the head of the team "Brain molecular imaging" included in the Inserm Unit 930 "Imaging and brain" in the University of Tours. This team associates the development of new fluor-18 labeled PET tracers, preclinical studies in animal models and clinical applications. The current research of Sylvie Chalon is focused on PET exploration of neurodegeneration and neuroinflammation in animal models of brain disorders such as Parkinson and Alzheimer's disease. She published more than 120 peer review papers in this field.

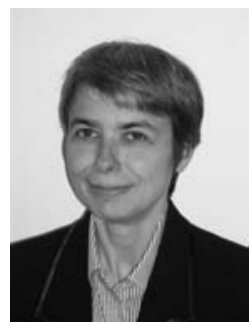

Irène Buvat received her Ph.D. degree in particle and nuclear physics from Paris Sud University, France, in 1992, and then spent one year at University College London, UK, and two years at the National Institutes of Health, Bethesda, USA. She entered the French Centre National de la Recherche Scientifique (CNRS) in 1995 and is currently the head of research at the Service Hospitalier Frédéric Joliot PET center, Orsay, France. Her research activities focus on developing correction and tomographic reconstruction methods in PET and SPECT to improve the accuracy and reduce the variability of measurements made from PET and SPECT images. She is currently the spokesperson of the worldwide OpenGATE collaboration developing the GATE Monte Carlo simulation tool dedicated to Monte Carlo simulations in emission and transmission tomography and radiotherapy. Irène Buvat is also largely involved into making quantification in SPECT and PET a clinical reality and contributed to many studies demonstrating the clinical values of quantification to improve image interpretation. She has authored or co-authored more than 110 peer-reviewed articles. She acts as an associate editor of IEEE Transactions on Medical Imaging and of IEEE Transactions on Nuclear Science and serves on the Editorial Board of the Journal of Nuclear Medicine and on the International Advisory Board of Physics in Medicine and Biology.

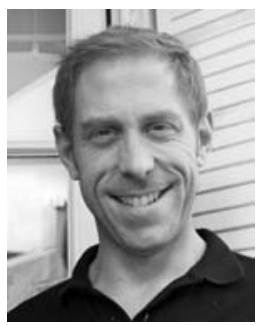

Clovis Tauber received the Ph.D. degree from the Institut National Polytechnique de Toulouse, France, in 2005. From 2005 to 2007 he was a Postdoctoral Research Fellow at the Institut de Recherche en Informatique de Toulouse (IRIT-ENSEEIHT), France. In 2008, he was a Postdoctoral Research Fellow at the Quantitative Image Analysis Unit, Institut Pasteur, France.

Since 2008, he is an Associate Professor at the University of Tours and he has joined the UMRS INSERM U930 Imaging and Brain Unit, Tours, France. $\mathrm{He}$ is responsible of the Medical Image Processing Group within the Brain Molecular Imaging team. His research interests are centered around medical image reconstruction, filtering, segmentation, registration and quantification, with a particular interest in exploiting the consistency of spatio-temporal information in dynamic image sequences. 